\title{
关于不定方程 $x^{4}+k x^{2} y^{2}+y^{4}=z^{2}$ 的可解性
}

\author{
郑 德 勋 \\ (四川大学数学系, 成都)
}

在四次不定方程早期的研究历史中, 方程

$$
x^{4}+k x^{2} y^{2}+y^{4}=z^{2}, x y \neq 0,
$$

曾扮演过重要的角色 ${ }^{[1,2]}$. 本文就方程 (1) 的可解性提供了一个新的判别法, 且于可解时可具 体地给出一个或多个互素的解来.

定理设 $k>2$, 且

$$
k-2=a^{\iota}+b^{2}, k+2=a_{1}^{2}+b_{1}^{2} .
$$

则当存在 $\left|\lambda_{1}\right|=\left|\lambda_{2}\right|=1$ 使

$$
\begin{gathered}
d_{1}=a+\lambda_{1} a_{1}, d_{2}=b+\lambda_{2} b_{1}, \\
d_{1}^{2}+d_{2}^{2}=d^{2}, d_{1} d_{2} \neq 0
\end{gathered}
$$

时,方程 (1)有解

$$
\left\{\begin{array}{l}
x=2 d^{\prime}\left|a d_{2}^{\prime}-b d_{1}^{\prime}\right|, \quad y=\left|\left(a d_{1}^{\prime}+b d_{2}^{\prime}\right)\left(\lambda_{1} \lambda_{2} a_{1} d_{1}^{\prime}+b_{1} d_{2}^{\prime}\right)\right|, \\
z=\frac{1}{4}\left|(k-2)\left(b_{1} d_{2}^{\prime}+\lambda_{1} \lambda_{2} a_{1} d_{1}^{\prime}\right)^{4}-(k+2)\left(b d_{2}^{\prime}+a d_{1}^{\prime}\right)^{4}\right|,
\end{array}\right.
$$

这里的 $d_{1}^{\prime}=\frac{d_{1}}{\left(d_{1}, d_{2}\right)}, d_{2}^{\prime}=\frac{d_{2}}{\left(d_{1}, d_{2}\right)}, d^{\prime}=\frac{d}{\left(d_{1}, d_{2}\right)}$.

证不难验证下式

$$
\begin{aligned}
& \left\{k\left(u^{2}-v^{2}\right)^{2}-2\left(u^{4}-v^{4}\right)\right\}^{2}+k\left\{k\left(u^{2}-v^{2}\right)^{2}-2\left(u^{4}-v^{4}\right)\right\}(2 u v)^{2}+(2 u v)^{4} \\
& \quad=\left\{(k-2) u^{4}-(k+2) v^{4}\right\}^{2},
\end{aligned}
$$

关于 $u, v$ 恒等成立. 故只须证明在定理所述条件下必能选取 $u v \neq 0$, 使得

$$
k\left(u^{2}-v^{2}\right)^{2}-2\left(u^{4}-v^{4}\right)=\left(u^{2}-v^{2}\right)\left[(k-2) u^{2}-(k+2) v^{2}\right]
$$

为一非零平方数即可. 为此又只需选取 $u v \neq 0$, 使

即

$$
\begin{gathered}
\left\{\begin{array}{l}
u^{2}-v^{2}=s^{2}, \\
(k-2) u^{2}-(k+2) v^{2}=t^{2},
\end{array} \text { stuv } \neq 0\right. \\
\left\{\begin{array}{l}
(k-2) s^{2}=t^{2}+4 v^{2}, \\
(k+2) s^{2}=t^{2}+4 u^{2},
\end{array} \text { stuv } \neq 0\right.
\end{gathered}
$$

成立即可. 而由假设 (2) 可知, 对任意整数 $\xi, \eta, \xi_{1}, \eta_{1}$ 和 $\varepsilon_{i}= \pm 1, i=1,2$,

$$
\begin{gathered}
s=\xi^{2}+\eta^{2}, t=a\left(\xi^{2}-\eta^{2}\right)-2 \varepsilon_{1} b \xi \eta, 2 v=b\left(\xi^{2}-\eta^{2}\right)+2 \varepsilon_{1} a \xi \eta ; \\
s=\xi_{1}^{2}+\eta_{1}^{2} t=a_{1}\left(\xi_{1}^{2}-\eta_{1}^{2}\right)-2 \varepsilon_{2} b_{1} \xi_{1} \eta_{1}, 2 u=b_{1}\left(\xi_{1}^{2}-\eta_{1}^{2}\right)+2 \varepsilon_{2} a_{1} \xi_{1} \eta_{1},
\end{gathered}
$$

均分别为 (7)、(8)式之解. 故只需证明在定理条件下必可选得 $\xi, \eta, \xi_{1}, \eta_{1}$ 和 $\varepsilon_{i}= \pm 1, i=1$, 2,3 , 使得 $s t u v \neq 0$, 且

本文 1986 年 3 月 10 日收到. 


$$
\left\{\begin{array}{l}
\xi^{2}+\eta^{2}=\xi_{1}^{2}+\eta_{1}^{2}, \\
a\left(\xi^{2}-\eta^{2}\right)-2 \varepsilon_{1} b \xi \eta=\varepsilon_{3}\left[a_{1}\left(\xi_{1}^{2}-\eta_{1}^{2}\right)-2 \varepsilon_{2} b_{1} \xi_{1} \eta_{1}\right]
\end{array}\right.
$$

成立即可. 为此, 我们取 $\xi_{1}=\xi, \eta_{1}=\eta$. 此时(11)式自然成立, 而(12)式变为

$$
f(\xi, \eta)=\left(a-\varepsilon_{3} a_{1}\right) \xi^{2}-\left(a-\varepsilon_{3} a_{1}\right) \eta^{2}-2 \varepsilon_{1}\left(b-\varepsilon_{1} \varepsilon_{2} \varepsilon_{3} b_{1}\right) \xi \eta=0 \text {. }
$$

再取 $\varepsilon_{3}=-\lambda_{1}, \varepsilon_{1}=1, \varepsilon_{2}=\lambda_{1} \lambda_{2}$, 则由假设(3)有 $f(\xi, \eta)=d_{1} \xi^{2}-2 d_{2} \xi \eta-d_{1} \eta^{2}=0$. 于是 由假设 (4) 可写 $d_{1} f(\xi, \eta)=\left(d_{1} \xi-d_{2} \eta\right)^{2}-d^{2} \eta^{2}$. 故 $f(\xi, \eta)=0$ 有解 $\xi=d_{2}-d=l, \eta=d_{1}$.

下证对如上选取之 $\xi, \eta, \xi_{1}, \eta_{1}, \varepsilon_{i}$ ，不仅(11)、(12)式同时成立，且有 $s t u v \neq 0$. 因此时由 (9)、(10)、(12)式给出

$$
\begin{gathered}
\left\{\begin{array}{l}
s=\xi^{2}+\eta^{2}=\left(d_{2}-d\right)^{2}+d_{1}^{2}=2 d\left(d-d_{2}\right)=-2 d l, \\
=a\left(l^{2}-d_{1}^{2}\right)-2 b l d_{1}=2 a d_{2} l-2 b l d_{1}=2 l\left(a d_{2}-b d_{1}\right),
\end{array}\right. \\
\left\{\begin{array}{l}
2 u=b_{1}\left(l^{2}-d_{1}^{2}\right)+2 \lambda_{1} \lambda_{2} a_{1} l d_{1}=2 l\left(b_{1} d_{2}+\lambda_{1} \lambda_{2} a_{1} d_{1}\right), \\
2 v=b\left(l^{2}-d_{1}^{2}\right)+2 a l d_{1}=2 l\left(b d_{2}+a d_{1}\right) .
\end{array}\right.
\end{gathered}
$$

而由假设(4)知 $d l \neq 0$, 故 $s \neq 0$. 再由(6)式知 $u \neq 0$. 又若 $t=0$, 则由(7)、(8)式应有

$$
(k-2) s^{2}=(2 v)^{2},(k+2) s^{2}=(2 u)^{2} \text {. }
$$

因 $s \neq 0$, 故必 $k-2=e^{2}, k+2=c^{2}$, 而这将导致 $c^{2}-e^{2}=4$, 从而 $e=0$, 从而 $k-$ 2 , 与定理之假设矛盾, 故有 $t \neq 0$. 最后, 如果 $\nu=0$, 则由 $l \neq 0$ 知

$$
0=b d_{2}+a d_{1}=b\left(b+\lambda_{2} b_{1}\right)+a\left(a+\lambda_{1} a_{1}\right)=k-2+\lambda_{1} a a_{1}+\lambda_{2} b b_{1} \text {. }
$$

进而应有

$$
\begin{aligned}
d_{1}^{2}+d_{2}^{2} & =\left(a+\lambda_{1} a_{1}\right)^{2}+\left(b+\lambda_{2} b_{1}\right)^{2} \\
& =2 k+2 \lambda_{1} a a_{1}+2 \lambda_{2} b b_{1}=2 k-2(k-2)=4,
\end{aligned}
$$

故必 $d_{1} d_{2}=0$, 而此与假设 (4)相矛盾. 因此 $v \neq 0$.

于是由(5)、(6)式知 (1) 式有非零解

$$
\begin{aligned}
& x=|s t|=4 d l^{2}\left|a d_{2}-b d_{1}\right|, \\
& y=|2 u v|=2 l^{2}\left|\left(b_{1} d_{2}+\lambda_{1} \lambda_{2} a_{1} d_{1}\right)\left(b d_{2}+a d_{1}\right)\right|, \\
& z=l^{4}\left|(k-2)\left(b_{1} d_{2}+\lambda_{1} \lambda_{2} a_{1} d_{1}\right)^{4}-(k+2)\left(b d_{2}+a d_{1}\right)^{4}\right| .
\end{aligned}
$$

再由方程 (1)的齐次性即得定理.

系 令 $t_{0}=1, t_{1}=7, t_{n+2}=6 t_{n+1}-t_{n}$. 则当 $n>0, k=\left(t_{n} \pm 1\right)^{2}+2$ 时, 方程 (1) 均可解.

证 因为此时有 $k-2=\left(t_{n} \pm 1\right)^{2}+0^{2}, k+2=\left(t_{n} \pm 1\right)^{2}+2^{2}$. 取 $a=t_{n} \pm 1, b=0$, $a_{1}=2, b_{1}=t_{n} \pm 1, \lambda_{1}=\mp 1, \lambda_{2}=1$, 则有 $d_{1}=t_{n} \pm 1 \mp 2=t_{n} \mp 1, d_{2}=t_{n} \pm 1$. 因 $n \geqslant 1$ 时有 $t_{n} \geqslant 7$, 故 $d_{1} d_{2} \neq 0$, 且 $\left.d_{1}^{2}+d_{2}^{2}=t_{n} \mp 1\right)^{2}+\left(t_{n} \pm 1\right)^{2}=2\left(t_{2}^{n}+1\right)$. 又因

$$
t_{n}=\frac{1}{2}\left(\varepsilon^{2 n+1}+\varepsilon^{2 n+1}\right), \varepsilon=1+\sqrt{2} \text {, 知有整数 } u_{n} \text { 使 } t_{n}^{2}-2 u_{n}^{2}=-1 \text {, }
$$

西而 $d_{1}^{2}+d_{2}^{2}=\left(2 u_{n}\right)^{2}=d^{2}$. 故由定理知方程(1)可解.

\section{考文献}

[1] Dickson, L. E., History of the Theory of Numbers, Vol. I1, Chelsea Publishing Company, New York, 1952. 634-638.

[ 2 ] Mordell, L. J., Diophanzine Equations, Academic Press, London and New York, 1969, 19-22. 